\title{
On the 2-Packing Differential of a Graph
}

\author{
A. Cabrera Martínez, M. L. Puertas@, and \\ J. A. Rodríguez-Velázquez
}

\begin{abstract}
Let $G$ be a graph of order $\mathrm{n}(G)$ and vertex set $V(G)$. Given a set $S \subseteq V(G)$, we define the external neighbourhood of $S$ as the set $N_{e}(S)$ of all vertices in $V(G) \backslash S$ having at least one neighbour in $S$. The differential of $S$ is defined to be $\partial(S)=\left|N_{e}(S)\right|-|S|$. In this paper, we introduce the study of the 2-packing differential of a graph, which we define as $\partial_{2 p}(G)=\max \{\partial(S): S \subseteq V(G)$ is a 2-packing $\}$. We show that the 2-packing differential is closely related to several graph parameters, including the packing number, the independent domination number, the total domination number, the perfect differential, and the unique response Roman domination number. In particular, we show that the theory of 2-packing differentials is an appropriate framework to investigate the unique response Roman domination number of a graph without the use of functions. Among other results, we obtain a Gallai-type theorem, which states that $\partial_{2 p}(G)+\mu_{R}(G)=\mathrm{n}(G)$, where $\mu_{R}(G)$ denotes the unique response Roman domination number of $G$. As a consequence of the study, we derive several combinatorial results on $\mu_{R}(G)$, and we show that the problem of finding this parameter is NP-hard. In addition, the particular case of lexicographic product graphs is discussed.
\end{abstract}

Mathematics Subject Classification. 05C69.

Keywords. Unique response Roman domination, 2-packing differential, differential of a graph .

\section{Introduction}

Given a graph $G=(V(G), E(G))$, the open neighbourhood of a vertex $v$ of a graph $G$ is defined to be $N(v)=\{u \in V(G): u$ is adjacent to $v\}$. The open neighbourhood of a set $S \subseteq V(G)$ is defined as $N(S)=\cup_{v \in S} N(v)$, while the 
external neighbourhood of $S$, or boundary of $S$, is defined as $N_{e}(S)=N(S) \backslash S$. The closed neighbourhood of $v$ is $N[v]=N(v) \cup\{v\}$ and the closed neighbourhood of a set $S \subseteq V(G)$ is $N[S]=N(S) \cup S$. We denote by $\operatorname{deg}(v)=|N(v)|$ the degree of vertex $v$, as well as $\delta(G)=\min _{v \in V(G)}\{\operatorname{deg}(v)\}$ the minimum degree of $G, \Delta(G)=\max _{v \in V(G)}\{\operatorname{deg}(v)\}$ the maximum degree of $G, \mathrm{n}(G)=|V(G)|$ the order of $G$ and $\mathrm{m}(G)=|E(G)|$ the size of $G$.

The differential of a set $S \subseteq V(G)$ is defined as $\partial(S)=\left|N_{e}(S)\right|-|S|$, while the differential of a graph $G$ is defined to be

$$
\partial(G)=\max \{\partial(S): S \subseteq V(G)\} .
$$

As described in [19], the definition of $\partial(G)$ was given by Hedetniemi about twenty-five years ago in an unpublished paper, and was also considered by Goddard and Henning [10]. After that, the differential of a graph has been studied by several authors, including $[2,5,19,24,26]$.

Lewis et al. [19] motivated the definition of differential from the following game, what we call graph differential game. "You are allowed to buy as many tokens as you like, say $k$ tokens, at a cost of one dollar each. You then place the tokens on some subset $D$ of $k$ vertices of a graph $G$. For each vertex of $G$ which has no token on it, but is adjacent to a vertex with a token on it, you receive one dollar. Your objective is to maximize your profit, that is, the total value received minus the cost of the tokens bought". Obviously, $\partial(D)=\left|N_{e}(D)\right|-|D|$ is the profit obtained with the placement $D$, while the maximum profit equals $\partial(G)$.

In order to introduce a variant of this game, we need the following terminology. A set $S \subseteq V(G)$ is a 2-packing if $N[u] \cap N[v]=\varnothing$ for every pair of different vertices $u, v \in S$. We define

$$
\wp(G)=\{S \subseteq V(G): S \text { is a 2-packing of } G\} .
$$

The 2-packing number of $G$, denoted by $\rho(G)$, is defined to be

$$
\rho(G)=\max \{|S|: S \in \wp(G)\} .
$$

Now, we consider a version of the graph differential game in which we impose two additional conditions: (1) every vertex which has no token on it is adjacent to at most one vertex with a token on it; and (2) no vertex with a token on it is adjacent to a vertex with a token on it. In this case, any placement $D$ of tokens is a 2-packing and so this version of the game can be called 2-packing graph differential game, as the maximum profit equals the 2-packing differential of $G$, which is defined as

$$
\partial_{2 p}(G)=\max \{\partial(S): S \in \wp(G)\} .
$$

We define a $\partial_{2 p}(G)$-set as a set $S \in \wp(G)$ with $\partial(S)=\partial_{2 p}(G)$. The same agreement will be assumed for optimal parameters associated to other characteristic sets defined in the paper.

Let $G$ be the graph shown in Fig. 1. In the graph differential game, if we place the tokens on vertices $a, b$ and $c$, then we obtain the maximum profit 


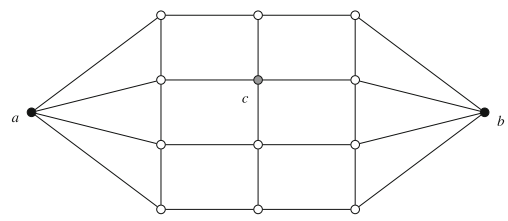

Figure 1. A graph $G$ with $\partial(G)=7$ and $\partial_{2 p}(G)=6$

$\partial(G)=7$. In contrast, in the 2-packing graph differential game, the maximum profit $\partial_{2 p}(G)=6$ is given by the placement of token on vertices $a$ and $b$.

In this paper we show that the 2-packing differential is closely related to several graph parameters, including the packing number, the independent domination number, the total domination number, the perfect differential, and the unique response Roman domination number. In particular, we show that the theory of 2-packing differentials is an appropriate framework to investigate the unique response Roman domination number of a graph without the use of functions.

The rest of the paper is organized as follows. Section 2 is devoted to provide some general bounds on the 2-packing differential, in terms of different parameters such as the maximum and minimum degrees of the graph, the number of vertices, the number of edges, among others. We show that the bounds are tight and, in some cases, we characterize the graphs achieving them. As a consequence of the study, we show that the problem of finding $\partial_{2 p}(G)$ is NP-hard. In Sect. 3 we obtain a Nordhaus-Gaddum type theorem for both the addition and the product of the 2-packing differential of a graph and its complement. Section 4 is devoted to the study of the relationship between the 2-packing differential and the perfect differential of a graph. In Sect. 5 we prove a Gallai-type theorem which states that $\partial_{2 p}(G)+\mu_{R}(G)=\mathrm{n}(G)$, where $\mu_{R}(G)$ denotes the unique response Roman domination number of $G$. We derive several consequences of this result, including the fact that the problem of finding $\mu_{R}(G)$ is NP-hard. We finally show the case of the lexicographic product graph in Sect. 6, where we obtain general lower and upper bounds of the 2-packing differential and the unique response Roman domination number. We also compute the exact value of both parameters for the lexicographic product of a path and any other graph.

\section{Basic Results and Computational Complexity}

In this section we present lower and upper bounds of the 2-packing differential of a graph. In some cases, we also characterize the graphs achieving such bounds. Moreover, these results will provide the computational complexity of the computation of the 2-packing differential. 


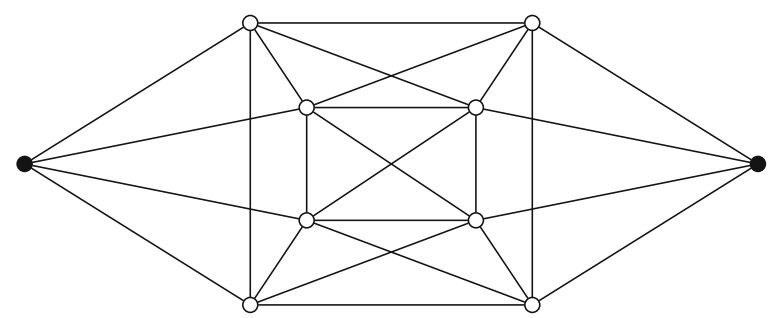

Figure 2. A graph $G$ with $\partial_{2 p}(G)=\rho(G)(\delta(G)-1)$

We begin with the following theorem that will be a key result in the rest of the paper. The bounds presented here are in terms of the maximum and minimum degrees of the graph and the 2-packing number.

Theorem 1. If $G$ is a graph with no isolated vertex, then the following statements hold.

(i) $\partial_{2 p}(G)=\max _{S \in \wp(G)}\left\{\sum_{v \in S}(\operatorname{deg}(v)-1)\right\}$.

(ii) If $G$ is a k-regular graph, then $\partial_{2 p}(G)=(k-1) \rho(G)$.

(iii) $\rho(G)(\delta(G)-1) \leq \partial_{2 p}(G) \leq \rho(G)(\Delta(G)-1)$.

(iv) $\partial_{2 p}(G)=\rho(G)(\Delta(G)-1)$ if and only if there exists a $\rho(G)$-set $S$ such that $\operatorname{deg}(v)=\Delta(G)$ for every $v \in S$.

(v) If $\partial_{2 p}(G)=\rho(G)(\delta(G)-1)$, then every vertex $v$ in every $\rho(G)$-set has degree $\operatorname{deg}(v)=\delta(G)$.

Proof. Notice that for any $S \in \wp(G)$, we have that $\partial(S)=|N(S)|-|S|=$ $\sum_{v \in S}(\operatorname{deg}(v)-1)$. Hence, $\partial_{2 p}(G)=\max _{S \in \wp(G)}\{\partial(S)\}=\max _{S \in \wp(G)}\{|N(S)|$ $-|S|\}=\max _{S \in \wp(G)}\left\{\sum_{v \in S}(\operatorname{deg}(v)-1)\right\}$. Therefore, (i) follows. From (i) we derive (ii) and (iii).

We proceed to prove (iv). If there exists a $\rho(G)$-set $S$ such that $\operatorname{deg}(v)=$ $\Delta(G)$ for every $v \in S$, then $\rho(G)(\Delta(G)-1)=\partial(S) \leq \partial_{2 p}(G)$. Hence, (iii) leads to $\partial_{2 p}(G)=\rho(G)(\Delta(G)-1)$. Conversely, if $\partial_{2 p}(G)=\rho(G)(\Delta(G)-1)$, then for any $\partial_{2 p}(G)$-set $D$ we have that $\rho(G)(\Delta(G)-1)=\partial(D)=\sum_{v \in D}(\operatorname{deg}(v)-1) \leq$ $\sum_{v \in D}(\Delta(G)-1) \leq|D|(\Delta(G)-1) \leq \rho(G)(\Delta(G)-1)$, which implies that $D$ is a $\rho(G)$-set and $\operatorname{deg}(v)=\Delta(G)$ for every $v \in D$.

Finally, we prove (v). Suppose on the contrary that there exists a $\rho(G)$-set $S^{\prime}$ and a vertex $v \in S^{\prime}$ such that $\operatorname{deg}(v)>\delta(G)$. Then, $\partial_{2 p}(G)=\max _{S \in \wp(}(G)$ $\left\{\sum_{v \in S}(\operatorname{deg}(v)-1)\right\} \geq \sum_{v \in S^{\prime}}(\operatorname{deg}(v)-1)>\rho(G)(\delta(G)-1)$. Figure 2 shows an example of a graph $G$ with $\partial_{2 p}(G)=\rho(G)(\delta(G)-1)$, the set of black-coloured vertices is the unique $\rho(G)$-set.

The first consequence of the preceding result is the determination of the complexity of the computation of the 2-packing differential. Given a graph $G$ and a positive integer $t$, the 2-packing problem is to decide whether there 
exists a 2-packing $S$ in $G$ such that $|S|$ is at least $t$. It is well known that the 2-packing problem is NP-complete. Hence, the optimization problem of finding $\rho(G)$ is NP-hard. Furthermore, it was shown in [17] that the 2-packing remains NP-complete for regular bipartite graphs. Therefore, from Theorem 1(ii) we derive the following result.

Corollary 1. The problem of finding the 2-packing differential of a graph is NP-hard, even for regular bipartite graphs.

As a second consequence of Theorem 1 we can derive the exact value of the 2-packing differential in particular graph families. To this end, we need the following definitions.

A set $S \subseteq V(G)$ of vertices is a dominating set if $N(v) \cap S \neq \varnothing$ for every vertex $v \in V(G) \backslash S$. Let $\mathcal{D}(G)$ be the set of dominating sets of $G$. The domination number of $G$ is defined to be,

$$
\gamma(G)=\min \{|S|: S \in \mathcal{D}(G)\} .
$$

Observe that $\gamma(G) \geq \rho(G)$ and $\mathcal{D}(G) \cap \wp(G) \neq \varnothing$ if and only if there exists a $\gamma(G)$-set which is a $\rho(G)$-set. A graph $G$ with $\mathcal{D}(G) \cap \wp(G) \neq \varnothing$ is called an efficient closed domination graph and in such case $\gamma(G)=\rho(G)$. Furthermore, Meir and Moon [21] showed that $\gamma(T)=\rho(T)$ for every tree $T$. Even so, there are trees in which $\mathcal{D}(T) \cap \wp(T)=\varnothing$.

If $G$ is a $k$-regular nonempty graph and there exists $S \in \mathcal{D}(G) \cap \wp(G)$, then $\mathrm{n}(G)=|S|+|N(S)|=|S|+|S| k=\rho(G)(1+k)$. Therefore, the following result is a direct consequence of Theorem 1 (ii).

Corollary 2. Let $k$ be a positive integer. If $G$ is a $k$-regular efficient closed domination graph, then

$$
\partial_{2 p}(G)=\frac{(k-1) \mathrm{n}(G)}{k+1}
$$

The next statement is a direct consequence of Theorem 1(i).

Corollary 3. For any graph $G$ of diameter at most two, $\partial_{2 p}(G)=\Delta(G)-1$.

We can also involve the order $\mathrm{n}(G)$ and the size $\mathrm{m}(G)$ of the graph and we obtain the following upper bound of the 2-packing differential.

Theorem 2. For any graph $G$ with no isolated vertex,

$$
\partial_{2 p}(G) \leq \rho(G)(\delta(G)-1)+2 \mathrm{~m}(G)-\mathrm{n}(G) \delta(G) .
$$

Furthermore, the equality holds if and only if there exists a $\rho(G)$-set $S$ such that $\operatorname{deg}(v)=\delta(G)$ for every $v \in V(G) \backslash S$. 


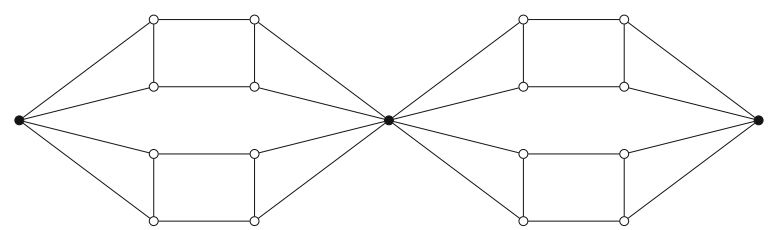

Figure 3. A graph $G$ with $\partial_{2 p}(G)=\rho(G)(\delta(G)-1)+$ $2 \mathrm{~m}(G)-\mathrm{n}(G) \delta(G)$. The set of black-coloured vertices forms a $\partial_{2 p}(G)$-set

Proof. If $S \subseteq V(G)$ is a $\partial_{2 p}(G)$-set, then

$$
\begin{aligned}
2 \mathrm{~m}(G) & =\sum_{v \in S} \operatorname{deg}(v)+\sum_{v \notin S} \operatorname{deg}(v) \\
& =\partial_{2 p}(G)+|S|+\sum_{v \notin S} \operatorname{deg}(v) \\
& \geq \partial_{2 p}(G)+|S|+(\mathrm{n}(G)-|S|) \delta(G) \\
& =\partial_{2 p}(G)+\mathrm{n}(G) \delta(G)+|S|(1-\delta(G)) \\
& \geq \partial_{2 p}(G)+\mathrm{n}(G) \delta(G)+\rho(G)(1-\delta(G)) .
\end{aligned}
$$

Therefore, the upper bound follows.

From the previous procedure we deduce that if $\partial_{2 p}(G)=2 \mathrm{~m}(G)-$ $\mathrm{n}(G) \delta(G)+(\delta(G)-1) \rho(G)$, then $|S|=\rho(G)$ and $\operatorname{deg}(v)=\delta(G)$ for every $v \in V(G) \backslash S$. Conversely, if $S$ is a $\rho(G)$-set and $\operatorname{deg}(v)=\delta(G)$ for every $v \in V(G) \backslash S$, then the same procedure leads to $2 \mathrm{~m}(G)-\mathrm{n}(G) \delta(G)+(\delta(G)-$ 1) $\rho(G)=\partial(S) \leq \partial_{2 p}(G)$, and by the upper bound we conclude that the equality holds.

Notice that for any $k$-regular graph, $2 \mathrm{~m}(G)=\mathrm{n}(G) \delta(G)=\mathrm{n}(G) k$, which implies that $\partial_{2 p}(G)=(k-1) \rho(G)=2 \mathrm{~m}(G)-\mathrm{n}(G) \delta(G)+(\delta(G)-1) \rho(G)$.

Figure 3 shows a graph $G$ with $\partial_{2 p}(G)=\rho(G)(\delta(G)-1)+2 \mathrm{~m}(G)-$ $\mathrm{n}(G) \delta(G)$. Observe that the set $S$ of black-coloured vertices forms a $\rho(G)$-set with $\operatorname{deg}(v)=\delta(G)$ for every $v \in V(G) \backslash S$.

The bound presented in Theorem 2 has a special behaviour in efficient closed domination graphs, as we show in the following two results.

Theorem 3. If $G$ is an efficient closed domination graph with no isolated vertex, then the following statements hold. 
(i) $\partial_{2 p}(G) \geq \mathrm{n}(G)-2 \rho(G)$.

(ii) If there exists a $\rho(G)$-set $S$ such that $\operatorname{deg}(v)=\delta(G)$ for every $v \in$ $V(G) \backslash S$, then

$$
\partial_{2 p}(G)=\mathrm{n}(G)-2 \rho(G) .
$$

Proof. For any set $D \in \mathcal{D}(G) \cap \wp(G)$,

$$
\partial_{2 p}(G) \geq \partial(D)=|N(D)|-|D|=|V(G) \backslash D|-|D|=\mathrm{n}(G)-2 \rho(G) .
$$

Now, in order to prove (ii), let $S$ be a $\rho(G)$-set such that $\operatorname{deg}(v)=\delta(G)$ for every $v \in V(G) \backslash S$. In the proof of Theorem 2 we have shown that $S$ is a $\partial_{2 p}(G)$-set. Notice that $|N(S)| \leq \mathrm{n}(G)-|S|=\mathrm{n}(G)-\rho(G)$. Hence, $\mathrm{n}(G)-2 \rho(G) \leq \partial_{2 p}(G)=\partial(S)=|N(S)|-\rho(G) \leq \mathrm{n}(G)-2 \rho(G)$. Therefore, $\partial_{2 p}(G)=\mathrm{n}(G)-2 \rho(G)$.

By Theorems 2 and 3 we deduce the following result.

Theorem 4. For any efficient closed domination graph $G$ with $\delta(G) \geq 1$,

$$
\rho(G) \geq\left\lceil\frac{\mathrm{n}(G)(\delta(G)+1)-2 \mathrm{~m}(G)}{\delta(G)+1}\right\rceil .
$$

Furthermore, the equality holds if and only if there exists a $\rho(G)$-set $S$ such that $\operatorname{deg}(v)=\delta(G)$ for every $v \in V(G) \backslash S$.

Proof. By combining the bounds given in Theorems 2 and 3, we deduce that $2 \mathrm{~m}(G)-\mathrm{n}(G) \delta(G)+(\delta(G)-1) \rho(G) \geq \partial_{2 p}(G) \geq \mathrm{n}(G)-2 \rho(G)$. Therefore, the bound follows.

Now, if $\rho(G)=\frac{\mathrm{n}(G)(\delta(G)+1)-2 \mathrm{~m}(G)}{\delta(G)+1}$, then we have equalities in the above inequality chain, and by Theorem 2 we conclude that there exists a $\rho(G)$-set $S$ such that $\operatorname{deg}(v)=\delta(G)$ for every $v \in V(G) \backslash S$.

Conversely, if there exists a $\rho(G)$-set $S$ such that $\operatorname{deg}(v)=\delta(G)$ for every $v \in V(G) \backslash S$, then Theorems 2 and 3 lead to $2 \mathrm{~m}(G)-\mathrm{n}(G) \delta(G)+(\delta(G)-$ 1) $\rho(G)=\mathrm{n}(G)-2 \rho(G)$, which implies that $\rho(G)=\frac{\mathrm{n}(G)(\delta(G)+1)-2 \mathrm{~m}(G)}{\delta(G)+1}$.

We now present lower and upper bounds of the 2-packing differential in terms of some dominating-type parameters. We begin with the following definitions.

A set $S \subseteq V(G)$ which is both dominating and independent is called an independent dominating set. Moreover, the independent domination number $i(G)$ is

$$
i(G)=\min \{|S|: S \text { is an independent dominating set }\} .
$$

On the other hand, let $S \subseteq V(G)$ be a $\partial_{2 p}(G)$-set, we define the partition of $V(G)$ associated to $S$ as $V(G)=N[S] \cup S^{\prime} \cup S^{\prime \prime}$, where $S^{\prime}=\{v \in$ $V(G) \backslash N[S]: N(v) \subseteq N(S)\}$ and $S^{\prime \prime}=V(G) \backslash\left(N[S] \cup S^{\prime}\right)$. Clearly $N[S], S^{\prime}, S^{\prime \prime}$ are pairwise disjoint sets and moreover, $V(G)=N[S] \cup S^{\prime} \cup S^{\prime \prime}$. We call $S^{\prime}$ and $S^{\prime \prime}$ the additional sets of the partition. 
Lemma 1. Let $G$ be a graph with no isolated vertex. Let $S \subseteq V(G)$ be a $\partial_{2 p}(G)$ set and let $S^{\prime}, S^{\prime \prime}$ the additional sets of the partition associated to $S$. The following statements hold.

(i) $S^{\prime}$ is an independent set.

(ii) $N(u) \cap\left(S^{\prime} \cup S^{\prime \prime}\right)=\varnothing$ for every $u \in S$.

(iii) $N(v) \cap S^{\prime \prime}=\varnothing$ for every $v \in S^{\prime}$.

(iv) If $|S|$ is maximum among all $\partial_{2 p}(G)$-sets then, $N(w) \cap N(S) \neq \varnothing$ for every $w \in S^{\prime \prime}$.

Proof. If there exist vertices $u, v \in S^{\prime}$ such that $u \in N(v)$, then $u \in N(S)$, by definition of $S^{\prime}$. This a contradiction with $u \in S^{\prime}$. If $x \in N(u) \cap\left(S^{\prime} \cup S^{\prime \prime}\right)$ for a vertex $u \in S$ then, $x \in N[S] \cap\left(S^{\prime} \cup S^{\prime \prime}\right)$, which is not possible by definition of the partition. In a similar way, if $y \in N(v) \cap S^{\prime \prime}$ for a vertex $v \in S^{\prime}$ then, $y \in N(S) \cap S^{\prime \prime}$, which is not possible by definition of $S^{\prime \prime}$. Finally, in order to prove (iv), suppose that there exists a vertex $w \in S^{\prime \prime}$ such that $N(w) \subseteq S^{\prime \prime}$. Notice that $S_{w}=S \cup\{w\}$ is a 2-packing of $G$ and that $\partial\left(S_{w}\right) \geq \partial(S)=\partial_{2 p}(G)$, which is a contradiction because $\left|S_{w}\right|>|S|$. Hence, for every $w \in S^{\prime \prime}$, it follows that $N(w) \cap N(S) \neq \varnothing$.

In the following theorem we present lower and upper bounds of the 2packing differential, and we characterize graphs attaining the upper one.

Theorem 5. For any graph $G$ with no isolated vertex,

$$
\Delta(G)-1 \leq \partial_{2 p}(G) \leq \frac{(\Delta(G)-1)(\mathrm{n}(G)-i(G))}{\Delta(G)} .
$$

Furthermore, the following statements are equivalent.

(i) $\partial_{2 p}(G)=\frac{(\Delta(G)-1)(\mathrm{n}(G)-i(G))}{\Delta(G)}$.

(ii) There exist an $i(G)$-set $D$ and a 2-packing $S \subseteq D$ such that $N(S) \cup D=$ $V(G)$ and $\operatorname{deg}(v)=\Delta(G)$ for every $v \in S$.

Proof. The lower bound is obtained by definition of $\partial_{2 p}(G)$. Let $S$ be a $\partial_{2 p}(G)$ set and let $S^{\prime}, S^{\prime \prime}$ be the additional sets of the partition associated to $S$. Let $S_{1}$ be an independent dominating set of the subgraph induced by $S^{\prime \prime}$ then, $\left|S_{1}\right| \leq\left|S^{\prime \prime}\right|$. Also, by using Lemma 1, the set $S \cup S^{\prime} \cup S_{1}$ is an independent dominating set of $G$. Hence,

$|N(S)|=\mathrm{n}(G)-\left(|S|+\left|S^{\prime}\right|+\left|S^{\prime \prime}\right|\right) \leq \mathrm{n}(G)-\left(|S|+\left|S^{\prime}\right|+\left|S_{1}\right|\right) \leq \mathrm{n}(G)-i(G)$.

Also, notice that $|N(S)| \leq \Delta(G)|S|$. Hence,

$$
\begin{aligned}
\Delta(G) \partial_{2 p}(G) & =\Delta(G)(|N(S)|-|S|) \\
& =\Delta(G)|N(S)|-\Delta(G)|S| \\
& \leq \Delta(G)|N(S)|-|N(S)| \\
& =(\Delta(G)-1)|N(S)| \\
& \leq(\Delta(G)-1)(\mathrm{n}(G)-i(G)) .
\end{aligned}
$$


Hence, the upper bound follows.

Now, if (i) holds, then we have equalities in the previous inequality chains. From these, we deduce that $S^{\prime \prime}=\varnothing, D=S \cup S^{\prime}$ is an $i(G)$-set, where $S$ is a 2-packing and $|N(S)|=\Delta(G)|S|=n-i(G)$, which implies that $N(S) \cup D=$ $V(G)$ and $\operatorname{deg}(v)=\Delta(G)$ for every $v \in S$. Therefore, (ii) follows.

Finally, if (ii) holds, i.e., there exist an $i(G)$-set $D$ and a 2-packing $W \subseteq D$ such that $N(W) \cup D=V(G)$ and $\operatorname{deg}(v)=\Delta(G)$ for every $v \in W$, then $\Delta(G)|W|=|N(W)|=\mathrm{n}(G)-i(G)$ and so

$$
\partial_{2 p}(G) \geq \partial(W)=|N(W)|-|W|=\mathrm{n}(G)-i(G)-\frac{\mathrm{n}(G)-i(G)}{\Delta(G)} .
$$

Therefore, from the upper bound we deduce that the equality holds.

Corollary 4. Let $G$ be a graph with no isolated vertex. If $\Delta(G)=\mathrm{n}(G)-i(G)$, then $\partial_{2 p}(G)=\Delta(G)-1$.

The converse of the result above does not hold. For instance, let $G$ be a graph having a vertex $v \in V(G)$ of maximum degree $\operatorname{deg}(v)=\Delta(G)=\mathrm{n}(G)-3$ such that the two vertices in $V(G) \backslash N[v]$ are adjacent. In this case, $i(G)=2$ and $\partial_{2 p}(G)=\mathrm{n}(G)-4=\Delta(G)-1$, while $\Delta(G)<\mathrm{n}(G)-i(G)$.

Next, we consider a particular case of the equivalence given in Theorem 5.

Theorem 6. For any graph $G$ with no isolated vertex,

$$
\partial_{2 p}(G) \leq \mathrm{n}(G)-i(G)-1 .
$$

Furthermore, the equality holds if and only if $\Delta(G)=\mathrm{n}(G)-i(G)$.

Proof. From Theorem 5 we deduce that $\mathrm{n}(G)-i(G) \geq \Delta(G)$ and $\partial_{2 p}(G) \leq$ $\mathrm{n}(G)-i(G)-\frac{\mathrm{n}(G)-i(G)}{\Delta(G)}$. Therefore, the upper bound follows.

Now, if $\Delta(G)=\mathrm{n}(G)-i(G)$, then from the bounds given in Theorem 5 we deduce that $\partial_{2 p}(G)=\mathrm{n}(G)-i(G)-1$. Conversely, if $\partial_{2 p}(G)=\mathrm{n}(G)-i(G)-1$, then by Theorem 5 we deduce that $\Delta(G)=\mathrm{n}(G)-i(G)$, which completes the proof.

We now characterize all trees attaining the lower bound presented in Theorem 5. To this end, we will use the following notation. Given a tree $T$, a vertex $v \in V(T)$ with $\operatorname{deg}(v)=\Delta(T)$ and any vertex $v_{i} \in N(v)$, denote by $T_{i}(v)$ the sub-tree of $T$ rooted in $v_{i}$ obtained from $T$ by removing the edge $\left\{v, v_{i}\right\}$. For each $i \in\{1, \ldots, \Delta(T)\}$ such that $T_{i}(v)$ is non-trivial, denote by $w_{i}$ the neighbour of $v_{i}$ in $T_{i}(v)$ with the largest degree.

Theorem 7. Let $T$ be a non-trivial tree. Then, $\partial_{2 p}(T)=\Delta(T)-1$ if and only if, for each vertex $v \in V(T)$ with $\operatorname{deg}(v)=\Delta(T)$, the following conditions hold.

(i) $\operatorname{ecc}(v) \leq 3$,

(ii) $\operatorname{deg}\left(v_{i}\right)+\sum_{j \neq i}\left(\operatorname{deg}\left(w_{j}\right)-1\right) \leq \Delta(T), \quad(i=1, \ldots, \Delta(T)$ and $j \neq i$ with $T_{j}(v)$ non-trivial), 


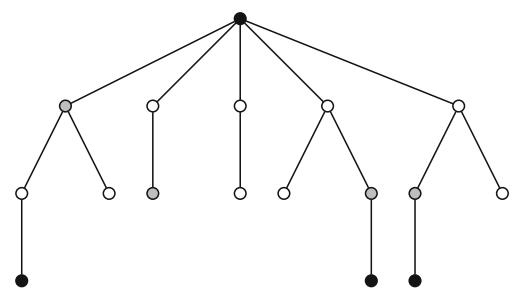

Figure 4. A tree with $\partial_{2 p}(T)=\Delta(T)-1=4$. The set of black-coloured vertices is a $\partial_{2 p}(T)$-set and the set of greycoloured vertices is another one

(iii) $\sum_{i}\left(\operatorname{deg}\left(w_{i}\right)-1\right) \leq \Delta(T)-1,\left(i \in\{1, \ldots, \Delta(T)\}\right.$ such that $T_{i}(v)$ is nontrivial).

Proof. Assume that $\partial_{2 p}(T)=\Delta(T)-1$ and take $v \in V(T)$ with $\operatorname{deg}(v)=$ $\Delta(T)$. The distance between two vertices $x, y \in V(T)$ will be denoted by $d(x, y)$.

If there exists $w \in V(T)$ such that $d(v, w)=4$, then for $u \in N(w)$ with $d(v, u)=3$ the set $\{v, u\}$ is a 2-packing and $\operatorname{deg}(u) \geq 2$. Thus, $\partial_{2 p}(T) \geq$ $\partial(\{v, u\}) \geq(\operatorname{deg}(u)-1)+(\operatorname{deg}(v)-1)>\operatorname{deg}(v)-1=\Delta(T)-1$, which is a contradiction. Therefore, $\operatorname{ecc}(v) \leq 3$.

The vertex set $X=\left\{v_{i}\right\} \cup\left\{w_{j}: j \neq i, T_{j}(v)\right.$ is non-trivial $\}$ is a 2-packing, so $\partial(X)=\left(\operatorname{deg}\left(v_{i}\right)-1\right)+\sum_{j \neq i}\left(\operatorname{deg}\left(w_{j}\right)-1\right) \leq \partial_{2 p}(T)=\Delta(T)-1$, that gives (ii). Analogously, the set $\left\{w_{i}: T_{i}(v)\right.$ is non-trivial $\}$ is a 2-packing and this gives (iii).

Now, let $T$ be a non-trivial tree satisfying (i), (ii) and (iii), let $S$ be a 2-packing of $T$ and let $v$ be a vertex with $\operatorname{deg}(v)=\Delta(T)$. Since $\partial_{2 p}(T) \geq$ $\Delta(T)-1$, we proceed to show that $\partial_{2 p}(T) \leq \Delta(T)-1$. To this end, we differentiate the following three cases.

Case 1: $v \in S$. If there exists $u \in S \backslash\{v\}$, then $d(u, v) \geq 3$ and condition (i) gives that $\operatorname{deg}(u)=1$ (see Fig. 4). Therefore, $\partial(S)=\sum_{w \in S}(\operatorname{deg}(w)-1)=$ $\operatorname{deg}(v)-1=\Delta(T)-1$.

Case 2: $v_{i} \in S$, for some $v_{i} \in N(v)$. Notice that $N[v] \cap S=\left\{v_{i}\right\}$ and, if there exists $u \in S \backslash\left\{v_{i}\right\}$, then $d\left(u, v_{i}\right) \geq 3$. In such a case, $u$ belongs to a non-trivial rooted sub-tree $T_{j}(v), j \neq i$ and $d(u, v) \geq 2$ (see Fig. 4). Clearly, $S \cap V\left(T_{j}(v)\right)=\{u\}$ and from (ii) we obtain $\partial(S)=\sum_{w \in S}(\operatorname{deg}(w)-1) \leq$ $\left(\operatorname{deg}\left(v_{i}\right)-1\right)+\sum_{j \neq i}\left(\operatorname{deg}\left(w_{j}\right)-1\right) \leq \Delta(T)-1$.

Case 3: $N[v] \cap S=\emptyset$. Then every vertex $u \in S$ satisfies $d(u, v) \geq 2$ and from (iii) we obtain $\partial(S)=\sum_{w \in S}(\operatorname{deg}(w)-1) \leq \sum_{i}\left(\operatorname{deg}\left(w_{i}\right)-1\right) \leq \Delta(T)-1$.

Some upper bounds of the 2-packing differential can be also expressed in terms of the total domination number. A set $S \subseteq V(G)$ is a total dominating set of a graph $G$ without isolated vertices if every vertex $v \in V(G)$ is adjacent 
to at least one vertex in $S$. Let $\mathcal{D}_{t}(G)$ be the set of total dominating sets of $G$. The total domination number of $G$ is defined to be,

$$
\gamma_{t}(G)=\min \left\{|S|: S \in \mathcal{D}_{t}(G)\right\} .
$$

By definition, $\mathcal{D}_{t}(G) \subseteq \mathcal{D}(G)$, so that $\gamma(G) \leq \gamma_{t}(G)$. Furthermore, $\gamma_{t}(G) \leq$ $2 \gamma(G)$.

Lemma 2. Let $G$ be a graph with no isolated vertex. Let $S$ be a $\partial_{2 p}(G)$-set such that $|S|$ is maximum among all $\partial_{2 p}(G)$-sets and let $S^{\prime}, S^{\prime \prime}$ be the additional sets of the partition associated to $S$. The following statements hold.

(i) $\gamma_{t}(G) \leq 2|S|+\left|S^{\prime}\right|+\left|S^{\prime \prime}\right|$.

(ii) If $\gamma_{t}(G)=2|S|+\left|S^{\prime}\right|+\left|S^{\prime \prime}\right|$, then $S^{\prime} \cup S^{\prime \prime}=\varnothing$.

Proof. By Lemma 1, we know that $N(w) \cap N(S) \neq \varnothing$ for every $w \in S^{\prime \prime}$. Now, we define $W^{\prime}$ as a set of minimum cardinality among the sets $W \subseteq N(S)$ satisfying that $N(x) \cap W \neq \varnothing$ for every $x \in S \cup S^{\prime} \cup S^{\prime \prime}$.

Notice that $S \cup W^{\prime}$ is a total dominating set of $G$, which implies that $\gamma_{t}(G) \leq|S|+\left|W^{\prime}\right| \leq 2|S|+\left|S^{\prime}\right|+\left|S^{\prime \prime}\right|$.

In order to prove (ii) assume $\gamma_{t}(G)=2|S|+\left|S^{\prime}\right|+\left|S^{\prime \prime}\right|$. In this case, $\left|W^{\prime}\right|=|S|+\left|S^{\prime}\right|+\left|S^{\prime \prime}\right|$. Suppose that there exists $x \in S^{\prime} \cup S^{\prime \prime}$. Since there exists $y \in N(x) \cap W^{\prime} \cap N(S)$, the minimality of $\left|W^{\prime}\right|$ leads to $\left|W^{\prime}\right|<|S|+\left|S^{\prime}\right|+\left|S^{\prime \prime}\right|$, which is a contradiction. Therefore, $S^{\prime} \cup S^{\prime \prime}=\varnothing$.

Theorem 8. For any graph $G$ with no isolated vertex,

$$
\partial_{2 p}(G) \leq \mathrm{n}(G)-\gamma_{t}(G) .
$$

Furthermore, the equality holds if and only if $G$ is an efficient closed domination graph with $\gamma_{t}(G)=2 \gamma(G)$.

Proof. Let $S$ be a $\partial_{2 p}(G)$-set such that $|S|$ is maximum among all $\partial_{2 p}(G)$ sets and let $S^{\prime}, S^{\prime \prime}$ be the additional sets of the partition associated to $S$. By Lemma $2, \gamma_{t}(G) \leq 2|S|+\left|S^{\prime}\right|+\left|S^{\prime \prime}\right|$. Hence,

$$
\partial_{2 p}(G)=|N(S)|-|S|=\mathrm{n}(G)-2|S|-\left|S^{\prime}\right|-\left|S^{\prime \prime}\right| \leq \mathrm{n}(G)-\gamma_{t}(G) .
$$

Therefore, the upper bound follows.

Now, if $\mathcal{D}(G) \cap \wp(G) \neq \varnothing$ and $\gamma_{t}(G)=2 \gamma(G)$, then from the upper bound above and the lower bound given in Theorem 3 we deduce that $\partial_{2 p}(G)=$ $\mathrm{n}(G)-\gamma_{t}(G)$. Conversely, if $\partial_{2 p}(G)=\mathrm{n}(G)-\gamma_{t}(G)$, then we have equalities in the above inequality chain. In particular, we deduce that $2|S|+\left|S^{\prime}\right|+\left|S^{\prime \prime}\right|=$ $\gamma_{t}(G)$, and Lemma 2 leads to $S^{\prime} \cup S^{\prime \prime}=\varnothing$. Hence, $S \in \mathcal{D}(G) \cap \wp(G)$ and so, $\gamma_{t}(G)=2|S|=2 \gamma(G)$, which completes the proof.

Theorem 9. For any graph $G$ with no isolated vertex,

$$
\partial_{2 p}(G) \leq \frac{2 \mathrm{~m}(G)+\rho(G)(\delta(G)-1)-\gamma_{t}(G) \delta(G)}{\delta(G)+1} .
$$

Furthermore, the equality holds if and only if $\gamma_{t}(G)=2 \gamma(G)$ and there exists a set $S \in \mathcal{D}(G) \cap \wp(G)$ such that $\operatorname{deg}(v)=\delta(G)$ for every $v \in V(G) \backslash S$. 
Proof. Let $S$ be a $\partial_{2 p}(G)$-set such that $|S|$ is maximum among all $\partial_{2 p}(G)$-sets, and let $m^{\prime}$ be the size of the subgraph of $G$ induced by $V(G) \backslash S$. Notice that every vertex in $N(S)$ has exactly one neighbour in $S$, which implies that

$$
m^{\prime}=\frac{1}{2}\left(\sum_{v \in N(S)}(\operatorname{deg}(v)-1)+\sum_{v \in V(G) \backslash N[S]} \operatorname{deg}(v)\right) .
$$

Observe that Lemma 2 leads to $\gamma_{t}(G) \leq 2|S|+|V(G) \backslash N[S]|$. Hence,

$$
\begin{aligned}
\mathrm{m}(G)= & \sum_{v \in S} \operatorname{deg}(v)+m^{\prime}=\left(\partial_{2 p}(G)+|S|\right)+\frac{1}{2} \\
& \times\left(\sum_{v \in N(S)}(\operatorname{deg}(v)-1)+\sum_{v \in V(G) \backslash N[S]} \operatorname{deg}(v)\right), \quad \text { by Theorem } 1(\mathrm{i}), \\
\geq & \partial_{2 p}(G)+|S|+\frac{1}{2}\left(|N(S)|(\delta(G)-1)+\left(\gamma_{t}(G)-2|S|\right) \delta(G)\right) \\
\geq & \partial_{2 p}(G)+\frac{1}{2}(|S|+(|S|-|N(S)|)+(|N(S)|-|S|) \delta(G) \\
& \left.+\gamma_{t}(G) \delta(G)-|S| \delta(G)\right) \\
= & \partial_{2 p}(G)+\frac{1}{2}\left(\partial_{2 p}(G)(\delta(G)-1)-(\delta(G)-1)|S|+\gamma_{t}(G) \delta(G)\right) \\
\geq & \partial_{2 p}(G)+\frac{1}{2}\left(\partial_{2 p}(G)(\delta(G)-1)-(\delta(G)-1) \rho(G)+\gamma_{t}(G) \delta(G)\right) .
\end{aligned}
$$

Therefore, the upper bound follows.

Now, if the bound is achieved, then from the previous procedure we deduce that $\operatorname{deg}(v)=\delta(G)$ for every $v \in V(G) \backslash S,|S|=\rho(G)$ and $\gamma_{t}(G)=$ $2|S|+|V(G) \backslash N[S]|=\mathrm{n}(G)-\partial_{2 p}(G)$. Thus, by Theorem 8 we conclude that $\mathcal{D}(G) \cap \wp(G) \neq \varnothing$ and $\gamma_{t}(G)=2 \gamma(G)$. Conversely, if $\gamma_{t}(G)=2 \gamma(G)$ and there exists a set $D \in \mathcal{D}(G) \cap \wp(G)$ such that $\operatorname{deg}(v)=\delta(G)$ for every $v \in V(G) \backslash D$, then the same procedure leads to

$2 \mathrm{~m}(G)+\rho(G)(\delta(G)-1)-\gamma_{t}(G) \delta(G)=(\delta(G)+1) \partial(D) \leq(\delta(G)+1) \partial_{2 p}(G)$,

and by the upper bound we conclude that the equality holds.

To conclude this section, we derive some bounds in terms of the order of $G$.

Theorem 10. The following statements hold for any graph $G$ with no isolated vertex.

(i) If $k \in\{1,2,3\}$ and $\Delta(G)=\mathrm{n}(G)-k$, then $\partial_{2 p}(G)=\mathrm{n}(G)-k-1$.

(ii) If $\Delta(G) \leq \mathrm{n}(G)-4$, then $\partial_{2 p}(G) \leq \mathrm{n}(G)-4$ and the equality holds if and only if $G$ is an efficient closed domination graph with $\gamma(G)=2$.

Proof. Assume $\Delta(G)=\mathrm{n}(G)-k$. If $k \in\{1,2\}$, then $\rho(G)=1$ and so $\partial_{2 p}(G)=$ $\Delta(G)-1=\mathrm{n}(G)-k-1$. Now, let $S$ be a $\partial_{2 p}(G)$-set. If $k=3$ then either 
$\rho(G)=1$, and we proceed as above, or $|S| \geq 2$, which implies that $\mathrm{n}(G)-4=$ $\Delta(G)-1 \leq \partial_{2 p}(G)=\partial(S)=|N(S)|-|S| \leq \mathrm{n}(G)-2|S| \leq \mathrm{n}(G)-4$. Therefore, (i) follows.

From now on, assume $\Delta(G) \leq \mathrm{n}(G)-4$. Let $S$ be a $\partial_{2 p}(G)$-set. If $|S|=1$, then $\partial_{2 p}(G)=\Delta(G)-1 \leq \mathrm{n}(G)-5$. Now, if $|S| \geq 2$, then $\partial_{2 p}(G)=\partial(S)=$ $|N(S)|-|S| \leq \mathrm{n}(G)-2|S| \leq \mathrm{n}(G)-4$. Therefore, the equality $\partial_{2 p}(G)=$ $\partial(S)=\mathrm{n}(G)-4$ holds if and only if $S \in \mathcal{D}(G) \cap \wp(G)$ and $|S|=2$.

\section{Nordhaus-Gaddum Type Relations}

Nordhaus and Gaddum [22] in 1956 proposed lower and upper bounds, in terms of the order of the graph, on the sum and the product of the chromatic number of a graph and its complement. Since then, several inequalities of a similar type have been proposed for other graph parameters. In this section we derive some Nordhaus-Gaddum type relations for the 2-packing differential.

A set $S \subseteq V(G)$ is an open packing if $N(u) \cap N(v)=\varnothing$ for every pair of different vertices $u, v \in S$. The open packing number of $G$, denoted by $\rho_{o}(G)$, is the maximum cardinality among all open packings of $G$. A graph $G$ is an efficient open domination graph if there exists an open packing which is a dominating set. Recent results about this topic can be found in $[15,18]$.

Notice that the complement of $G$, denoted by $G^{c}$, is an efficient closed domination graph with $\gamma\left(G^{c}\right)=2$ if and only if $G$ is an efficient open domination graph with $\rho_{o}(G)=2$.

By Theorem 10 and the lower bound given in Theorem 5, we deduce the following result.

Theorem 11. Given a graph $G$, the following statements hold.

- If $\Delta(G)=\mathrm{n}(G)-2$ and $\delta(G)=1$, then $\partial_{2 p}(G)+\partial_{2 p}\left(G^{c}\right)=2 \mathrm{n}(G)-6$ and $\partial_{2 p}(G) \cdot \partial_{2 p}\left(G^{c}\right)=(\mathrm{n}(G)-3)^{2}$.

- If $\Delta(G)=\mathrm{n}(G)-2$ and $\delta(G)=2$, then $\partial_{2 p}(G)+\partial_{2 p}\left(G^{c}\right)=2 \mathrm{n}(G)-7$ and $\partial_{2 p}(G) \cdot \partial_{2 p}\left(G^{c}\right)=(\mathrm{n}(G)-3)(\mathrm{n}(G)-4)$.

- If $\Delta(G)=\mathrm{n}(G)-3$ and $\delta(G)=1$, then $\partial_{2 p}(G)+\partial_{2 p}\left(G^{c}\right)=2 \mathrm{n}(G)-7$ and $\partial_{2 p}(G) \cdot \partial_{2 p}\left(G^{c}\right)=(\mathrm{n}(G)-3)(\mathrm{n}(G)-4)$.

- If $\Delta(G)=\mathrm{n}(G)-3$ and $\delta(G)=2$, then $\partial_{2 p}(G)+\partial_{2 p}\left(G^{c}\right)=2 \mathrm{n}(G)-8$ and $\partial_{2 p}(G) \cdot \partial_{2 p}\left(G^{c}\right)=(\mathrm{n}(G)-4)^{2}$.

- If $\Delta(G)=\mathrm{n}(G)-2$ and $\delta(G) \geq 3$, then

$2 \mathrm{n}(G)-\delta(G)-5 \leq \partial_{2 p}(G)+\partial_{2 p}\left(G^{c}\right) \leq 2 \mathrm{n}(G)-7$, $(\mathrm{n}(G)-3)(\mathrm{n}(G)-\delta(G)-2) \leq \partial_{2 p}(G) \cdot \partial_{2 p}\left(G^{c}\right) \leq(\mathrm{n}(G)-3)(\mathrm{n}(G)-4)$.

- If $\Delta(G)=\mathrm{n}(G)-2$ and $\delta(G) \geq 3$, then $\partial_{2 p}(G)+\partial_{2 p}\left(G^{c}\right)=2 \mathrm{n}(G)-7$ if and only if $\partial_{2 p}(G) \cdot \partial_{2 p}\left(G^{c}\right)=(\mathrm{n}(G)-3)(\mathrm{n}(G)-4)$ if and only if $G$ is an efficient open domination graph with $\rho_{o}(G)=2$. 
- If $\Delta(G)=\mathrm{n}(G)-3$ and $\delta(G) \geq 3$, then

$$
\begin{aligned}
& 2 \mathrm{n}(G)-\delta(G)-6 \leq \partial_{2 p}(G)+\partial_{2 p}\left(G^{c}\right) \leq 2 \mathrm{n}(G)-8, \\
& \quad(\mathrm{n}(G)-4)(\mathrm{n}(G)-\delta(G)-2) \leq \partial_{2 p}(G) \cdot \partial_{2 p}\left(G^{c}\right) \leq(\mathrm{n}(G)-4)^{2} .
\end{aligned}
$$

- If $\Delta(G)=\mathrm{n}(G)-3$ and $\delta(G) \geq 3$, then $\partial_{2 p}(G)+\partial_{2 p}\left(G^{c}\right)=2 \mathrm{n}(G)-8$ if and only if $\partial_{2 p}(G) \cdot \partial_{2 p}\left(G^{c}\right)=(\mathrm{n}(G)-4)^{2}$ if and only if $G$ is an efficient open domination graph with $\rho_{o}(G)=2$.

- If $\Delta(G) \leq \mathrm{n}(G)-4$ and $\delta(G)=1$, then

$$
\begin{aligned}
& \mathrm{n}(G)+\Delta(G)-4 \leq \partial_{2 p}(G)+\partial_{2 p}\left(G^{c}\right) \leq 2 \mathrm{n}(G)-7, \\
& \quad(\Delta(G)-1)(\mathrm{n}(G)-3) \leq \partial_{2 p}(G) \cdot \partial_{2 p}\left(G^{c}\right) \leq(\mathrm{n}(G)-3)(\mathrm{n}(G)-4) .
\end{aligned}
$$

- If $\Delta(G) \leq \mathrm{n}(G)-4$ and $\delta(G)=1$, then $\partial_{2 p}(G)+\partial_{2 p}\left(G^{c}\right)=2 \mathrm{n}(G)-7$ if and only if $\partial_{2 p}(G) \cdot \partial_{2 p}\left(G^{c}\right)=(\mathrm{n}(G)-4)(\mathrm{n}(G)-3)$ if and only if $G$ is an efficient closed domination graph with $\gamma(G)=2$.

- If $\Delta(G) \leq \mathrm{n}(G)-4$ and $\delta(G)=2$, then

$$
\begin{aligned}
& \mathrm{n}(G)+\Delta(G)-5 \leq \partial_{2 p}(G)+\partial_{2 p}\left(G^{c}\right) \leq 2 \mathrm{n}(G)-8, \\
& \quad(\Delta(G)-1)(\mathrm{n}(G)-4) \leq \partial_{2 p}(G) \cdot \partial_{2 p}\left(G^{c}\right) \leq(\mathrm{n}(G)-4)^{2} .
\end{aligned}
$$

- If $\Delta(G) \leq \mathrm{n}(G)-4$ and $\delta(G)=2$, then $\partial_{2 p}(G)+\partial_{2 p}\left(G^{c}\right)=2 \mathrm{n}(G)-8$ if and only if $\partial_{2 p}(G) \cdot \partial_{2 p}\left(G^{c}\right)=(\mathrm{n}(G)-4)^{2}$ if and only if $G$ is an efficient closed domination graph with $\gamma(G)=2$.

- If $\Delta(G) \leq \mathrm{n}(G)-4$ and $\delta(G) \geq 3$, then

$$
\begin{aligned}
& \mathrm{n}(G)+\Delta(G)-\delta(G)-3 \leq \partial_{2 p}(G)+\partial_{2 p}\left(G^{c}\right) \leq 2 \mathrm{n}(G)-8 . \\
& \quad(\Delta(G)-1)(\mathrm{n}(G)-\delta(G)-2) \leq \partial_{2 p}(G) \cdot \partial_{2 p}\left(G^{c}\right) \leq(\mathrm{n}(G)-4)^{2} .
\end{aligned}
$$

- If $\Delta(G) \leq \mathrm{n}(G)-4$ and $\delta(G) \geq 3$, then $\partial_{2 p}(G)+\partial_{2 p}\left(G^{c}\right)=2 \mathrm{n}(G)-8$ if and only if $\partial_{2 p}(G) \cdot \partial_{2 p}\left(G^{c}\right)=(\mathrm{n}(G)-4)^{2}$ if and only if $G$ is simultaneously an efficient closed domination and an efficient open domination graph with $\rho_{o}(G)=\gamma(G)=2$.

Proof. We will show the proofs of two typical items and the remaining ones have similar proofs.

Assume that $\Delta(G)=\mathrm{n}(G)-2$ and $\delta(G)=1$ then, $\Delta\left(G^{c}\right)=\mathrm{n}\left(G^{c}\right)-1-$ $\delta(G)=\mathrm{n}(G)-2$ and Theorem 10 gives $\partial_{2 p}(G)=\mathrm{n}(G)-3, \partial_{2 p}\left(G^{c}\right)=\mathrm{n}\left(G^{c}\right)-$ $3=\mathrm{n}(G)-3$. Therefore, $\partial_{2 p}(G)+\partial_{2 p}\left(G^{c}\right)=2 \mathrm{n}(G)-6$ and $\partial_{2 p}(G) \cdot \partial_{2 p}\left(G^{c}\right)=$ $(\mathrm{n}(G)-3)^{2}$.

Assume now that $\Delta(G)=\mathrm{n}(G)-2$ and $\delta(G) \geq 3$ then, we know that $\Delta\left(G^{c}\right)=\mathrm{n}\left(G^{c}\right)-1-\delta(G)=\mathrm{n}(G)-1-\delta(G) \leq \mathrm{n}(G)-4$. Theorem 5 gives $\Delta(G)-1+\Delta\left(G^{c}\right)-1=2 \mathrm{n}(G)-\delta(G)-5 \leq \partial_{2 p}(G)+\partial_{2 p}\left(G^{c}\right)$ and moreover, $(\Delta(G)-1) \cdot\left(\Delta\left(G^{c}\right)-1\right)=(\mathrm{n}(G)-3)(\mathrm{n}(G)-\delta(G)-2) \leq \partial_{2 p}(G) \cdot \partial_{2 p}\left(G^{c}\right)$. On the other hand, by Theorem 10, we obtain that $\partial_{2 p}(G)=\mathrm{n}(G)-3$ and $\partial_{2 p}\left(G^{c}\right) \leq \mathrm{n}\left(G^{c}\right)-4=\mathrm{n}(G)-4$. This gives the desired upper bounds $\partial_{2 p}(G)+$ $\partial_{2 p}\left(G^{c}\right) \leq 2 \mathrm{n}(G)-7$ and $\partial_{2 p}(G) \cdot \partial_{2 p}\left(G^{c}\right) \leq(\mathrm{n}(G)-3)(\mathrm{n}(G)-4)$. 
Finally, if $\Delta(G)=\mathrm{n}(G)-2$ and $\delta(G) \geq 3$, then $\partial_{2 p}(G)+\partial_{2 p}\left(G^{c}\right)=$ $2 \mathrm{n}(G)-7$ if and only if $\partial_{2 p}\left(G^{c}\right)=\mathrm{n}(G)-4$ if and only if $G^{c}$ is an efficient closed domination graph with $\gamma\left(G^{c}\right)=2$ if and only if $G$ is an efficient open domination graph with $\rho_{o}(G)=2$. Moreover, $\partial_{2 p}(G) \cdot \partial_{2 p}\left(G^{c}\right)=(\mathrm{n}(G)-$ $3)(\mathrm{n}(G)-4)$ if and only if $\partial_{2 p}\left(G^{c}\right)=\mathrm{n}(G)-4$, and this completes the proof of this item.

\section{Perfect Differential Versus 2-Packing Differential}

In this section we show some relationships between the 2-packing differential and the perfect differential of a graph. Given a set $S \subseteq V(G)$ and a vertex $v \in$ $S$, the external private neighbourhood $\operatorname{epn}(v, S)$ of $v$ with respect to $S$ is defined to be epn $(v, S)=\{u \in V(G) \backslash S: N(u) \cap S=\{v\}\}$. The perfect neighbourhood of a set $S \subseteq V(G)$ is defined to be $N_{p}(S)=\{v \in V(G) \backslash S:|N(v) \cap S|=1\}$. We define the perfect differential of a set $S \subseteq V(G)$ as $\partial_{p}(S)=\left|N_{p}(S)\right|-|S|$. The perfect differential of a graph, introduced by Cabrera Martínez and RodríguezVelázquez in [5], is defined as

$$
\partial_{p}(G)=\max \left\{\partial_{p}(S): S \subseteq V(G)\right\} .
$$

Now, $S \subseteq V(G)$ is a perfect dominating set of $G$ if every vertex in $V(G) \backslash S$ is adjacent to exactly one vertex in $S$. Let $\mathcal{D}^{p}(G)$ be the set of perfect dominating sets of $G$, note that the hole vertex set $V(G)$ is a perfect dominating set so, $\mathcal{D}^{p}(G) \neq \varnothing$. The perfect domination number of $G$ is defined to be,

$$
\gamma^{p}(G)=\min \left\{|S|: S \in \mathcal{D}^{p}(G)\right\} .
$$

Notice that $\mathcal{D}^{p}(G) \subseteq \mathcal{D}(G)$, which implies that $\gamma(G) \leq \gamma^{p}(G)$.

Theorem 12. [5] Given a nontrivial graph $G$, the following inequality chain holds.

$$
\mathrm{n}(G)-2 \gamma^{p}(G) \leq \partial_{p}(G) \leq \frac{\mathrm{n}(G)(\Delta(G)-1)}{\Delta(G)+1} .
$$

Furthermore, $\partial_{p}(G)=\frac{\mathrm{n}(G)(\Delta(G)-1)}{\Delta(G)+1}$ if and only if $\gamma^{p}(G)=\frac{\mathrm{n}(G)}{\Delta(G)+1}$.

The following theorems show some relationships between the 2-packing differential and the perfect differential.

Theorem 13. For any nontrivial graph $G$, the following statements hold.

(i) $\partial_{2 p}(G) \leq \partial_{p}(G)$.

(ii) $\partial_{2 p}(G) \leq \frac{\mathrm{n}(G)(\Delta(G)-1)}{\Delta(G)+1}$.

(iii) $\partial_{2 p}(G)=\frac{\mathrm{n}(G)(\Delta(G)-1)}{\Delta(G)+1}$ if and only if $\gamma^{p}(G)=\frac{\mathrm{n}(G)}{\Delta(G)+1}$.

Proof. Let $S$ be a $\partial_{2 p}(G)$-set. Since $S$ is a 2-packing, $N(S)=N_{p}(S)$. Hence, $\partial_{2 p}(G)=\partial(S)=|N(S)|-|S|=\left|N_{p}(S)\right|-|S|=\partial_{p}(S) \leq \partial_{p}(G)$. Therefore, (i) follows. 
Notice that (ii) is a direct consequence of (i) and Theorem 12 . We proceed to prove (iii). It is known that $\gamma^{p}(G) \geq \gamma(G) \geq \frac{\mathrm{n}(G)}{\Delta(G)+1}$ and $\gamma(G)=\frac{\mathrm{n}(G)}{\Delta(G)+1}$ if and only if there exists a $\gamma(G)$-set $S$ which is a $\rho(G)$-set and every vertex in $S$ has degree $\Delta(G)$, for instance, see [12]. Hence, by Theorem 1 , if $\gamma^{p}(G)=$ $\frac{\mathrm{n}(G)}{\Delta(G)+1}$, then

$$
\partial_{2 p}(G)=\max _{S \in \wp(G)}\left\{\sum_{v \in S}(\operatorname{deg}(v)-1)\right\}=\rho(G)(\Delta(G)-1)=\frac{\mathrm{n}(G)(\Delta(G)-1)}{\Delta(G)+1} .
$$

Conversely, if $\partial_{2 p}(G)=\frac{\mathrm{n}(G)(\Delta(G)-1)}{\Delta(G)+1}$, then by (i) and Theorem 12 we have that $\partial_{p}(G)=\frac{\mathrm{n}(G)(\Delta(G)-1)}{\Delta(G)+1}$. Therefore, Theorem 12 leads to $\gamma^{p}(G)=\frac{\mathrm{n}(G)}{\Delta(G)+1}$.

The difference $\partial_{p}(G)-\partial_{2 p}(G)$ can be as large as desired. Consider the graph obtained by attaching to each vertex of a 5-cycle a set of $k$ independent vertices. The resulting graph $G$ has order $\mathrm{n}(G)=5 k+5$ and satisfies $\partial_{p}(G)=$ $5 k-5$ (the set of vertices of the 5 -cycle is a $\partial_{p}(G)$-set) and $\partial_{2 p}(G)=k+1$ (each single vertex in the 5 -cycle is a $\partial_{2 p}(G)$-set). Moreover, the set of vertices of the 5 -cycle is a minimum perfect dominating set, so $\gamma^{p}(G)=5$ and $\partial_{p}(G)=$ $\mathrm{n}(G)-2 \gamma^{p}(G)$ takes the smallest possible value.

Regarding the case in which both parameters agree, we have the following result.

Theorem 14. For any graph $G$, at least one of the following statements hold.

(i) $\partial_{2 p}(G)=\partial_{p}(G)$.

(ii) $\partial_{2 p}(G) \geq \frac{1}{2}\left(\Delta(G) \partial_{p}(G)+2-(\mathrm{n}(G)-2)(\Delta(G)-2)\right)$.

Proof. By Theorem 13 we have that $\partial_{2 p}(G) \leq \partial_{p}(G)$. From now on, we assume that $\partial_{2 p}(G)<\partial_{p}(G)$. Let $S$ be a $\partial_{p}(G)$-set. Since $\partial_{p}(G)=\left|N_{p}(S)\right|-|S|$ and $\left|N_{p}(S)\right|+|S| \leq \mathrm{n}(G)$, it follows that $|S| \leq\left(\mathrm{n}(G)-\partial_{p}(G)\right) / 2$. Let $S_{1} \subseteq S$ be maximal 2-packing and $S_{2}=S \backslash S_{1}$. Observe that $S$ is not a 2-packing, as $\partial_{2 p}(G)<\partial_{p}(G)=\partial(S)$. Hence, $N\left[S_{2}\right] \cap N\left(S_{1}\right) \neq \varnothing$, which implies that

$\left|N_{p}(S)\right|=\left|N\left(S_{1}\right) \backslash N\left[S_{2}\right]\right|+\sum_{x \in S_{2}}|\operatorname{epn}(x, S)| \leq\left(\left|N\left(S_{1}\right)\right|-1\right)+\left|S_{2}\right|(\Delta(G)-1)$.

Thus,

$$
\begin{aligned}
\partial_{p}(G) & =\left|N_{p}(S)\right|-|S| \\
& \leq\left(\left|N\left(S_{1}\right)\right|-1\right)+\left|S_{2}\right|(\Delta(G)-1)-|S| \\
& =\partial\left(S_{1}\right)+\left|S_{1}\right|-1+\left|S_{2}\right|(\Delta(G)-1)-|S| \\
& =\partial\left(S_{1}\right)-1+\left|S_{2}\right|(\Delta(G)-2)
\end{aligned}
$$




$$
\begin{aligned}
& \leq \partial_{2 p}(G)-1+(|S|-1)(\Delta(G)-2) \\
& \leq \partial_{2 p}(G)-1+\left(\frac{\mathrm{n}(G)-\partial_{p}(G)}{2}-1\right)(\Delta(G)-2) .
\end{aligned}
$$

Therefore, the result follows.

The bound above is tight. For instance, for any integer $t \geq 2$, the double star $S_{t, t}$ satisfies that $\partial_{p}\left(S_{t, t}\right)=2 t-2, \partial_{2 p}\left(S_{t, t}\right)=t, \mathrm{n}\left(S_{t, t}\right)=2 t+2$ and $\Delta\left(S_{t, t}\right)=t+1$. Hence, the bound is achieved by any double star $S_{t, t}$ with $t \geq 2$.

\section{Unique Response Roman Domination Versus 2-Packing Differential}

In this section we establish a Gallai-type theorem which states the relationship between the 2-packing differential and the unique response Roman domination number.

Cockayne, Hedetniemi and Hedetniemi [7] defined a Roman dominating function on a graph $G$ to be a function $f: V(G) \longrightarrow\{0,1,2\}$ satisfying the condition that every vertex $u$ for which $f(u)=0$ is adjacent to at least one vertex $v$ for which $f(v)=2$. For $X \subseteq V(G)$ we define the weight of $X$ as $f(X)=\sum_{v \in X} f(v)$. The weight of $f$ is defined to be

$$
\omega(f)=f(V(G)) .
$$

The unique response version of Roman domination was introduced by Rubalcaba and Slater in [25] and studied further in [9,14,31]. A function $f$ : $V(G) \longrightarrow\{0,1,2\}$ with the sets $V_{0}, V_{1}, V_{2}$, where $V_{i}=\{v \in V(G): f(v)=i\}$ for $i \in\{0,1,2\}$, is a unique response Roman dominating function if $x \in V_{0}$ implies $\left|N(x) \cap V_{2}\right|=1$ and $x \in V_{1} \cup V_{2}$ implies that $N(x) \cap V_{2}=\varnothing$. The unique response Roman domination number of $G$, denoted by $\mu_{R}(G)$, is the minimum weight among all unique response Roman dominating functions on $G$.

Theorem 15. (Gallai-type theorem) For any graph $G$,

$$
\mu_{R}(G)+\partial_{2 p}(G)=\mathrm{n}(G) .
$$

Proof. It was shown in [9] that $\mu_{R}(G)=\min _{S \subseteq \wp(G)}\{2|S|+|V(G) \backslash(N(S) \cup S)|\}$. Hence, the result is deduced as follows.

$$
\begin{aligned}
\mu_{R}(G) & =\min _{S \subseteq \wp(G)}\{2|S|+|V(G) \backslash(N(S) \cup S)|\} \\
& =\min _{S \subseteq \wp(G)}\{2|S|+\mathrm{n}(G)-(|N(S)|+|S|) \mid\} \\
& =\min _{S \subseteq \wp(G)}\{\mathrm{n}(G)-\partial(S)\} \\
& =\mathrm{n}(G)-\max _{S \subseteq \wp(G)}\{\partial(S)\}
\end{aligned}
$$




$$
=\mathrm{n}(G)-\partial_{2 p}(G) \text {. }
$$

Theorem 15 allows us to derive results on the unique response Roman domination number from results on the 2-packing differential and vice versa. For instance, from Corollary 1 and Theorem 15 we deduce the following result.

Theorem 16. The problem of finding the unique response Roman domination number of a graph is NP-hard, even for regular bipartite graphs.

Theorem 15 suggests the challenge of obtaining new results on the unique response Roman domination number from the approach of 2-packing differentials. As an example, the following table summarizes some of those results obtained here. The first column describes the result that combined with Theorem 15 leads to the result on the second column.

From Result for graphs with no isolated vertex

Theorem 1(iii) $\quad \mu_{R}(G) \geq \mathrm{n}(G)-\rho(G)(\Delta(G)-1)$.

The equality holds if and only if there exists a $\rho(G)$-set $S$ such that $\operatorname{deg}(v)=\Delta(G)$ for every $v \in S$.

Theorem $2 \quad \mu_{R}(G) \geq \mathrm{n}(G)(\delta(G)+1)-\rho(G)(\delta(G)-1)-2 \mathrm{~m}(G)$.

The equality holds if and only if there exists a $\rho(G)$-set $S$ such that $\operatorname{deg}(v)=\delta(G)$ for every $v \in V(G) \backslash S$.

Theorem 3 If $G$ is an efficient closed domination graph, then $\mu_{R}(G) \leq 2 \rho(G)$. In particular, if there exists a $\rho(G)$-set $S$ such that $\operatorname{deg}(v)=\delta(G)$ for every $v \in V(G) \backslash S$, then $\mu_{R}(G)=2 \rho(G)$.

Theorem $5 \quad \mu_{R}(G) \geq \frac{\mathrm{n}(G)+(\Delta(G)-1) i(G)}{\Delta(G)}$.

Theorem $6 \quad \mu_{R}(G) \geq i(G)+1$.

The equality holds if and only if $\Delta(G)=\mathrm{n}(G)-i(G)$.

Theorem $8 \quad \mu_{R}(G) \geq \gamma_{t}(G)$.

The equality holds if and only if $G$ is an efficient closed domination graph and $\gamma_{t}(G)=2 \gamma(G)$.

Theorem 9 $\mu_{R}(G) \geq \frac{\mathrm{n}(G)(\delta(G)+1)-2 \mathrm{~m}(G)-\rho(G)(\delta(G)-1)+\gamma_{t}(G) \delta(G)}{\delta(G)+1}$.

The equality holds if and only if there exists a set $S \in \mathcal{D}(G) \cap \wp(G)$ such that $\operatorname{deg}(v)=\delta(G)$ for every $v \in V(G) \backslash S$.

Theorem 13(i) $\quad \mu_{R}(G) \geq \mathrm{n}(G)-\partial_{p}(G)$.

Theorem 13(ii) $\quad \mu_{R}(G)=\frac{2 \mathrm{n}(G)}{\Delta(G)+1}$ if and only if $\gamma^{p}(G)=\frac{\mathrm{n}(G)}{\Delta(G)+1}$. 


\section{The Case of Lexicographic Product Graphs}

In this final section we present the behaviour of the 2-packing differential under the lexicographic product operation. We first recall the following definition. Let $G$ and $H$ be two graphs. The lexicographic product of $G$ and $H$ is the graph $G \circ H$ whose vertex set is $V(G \circ H)=V(G) \times V(H)$ and $(u, v)(x, y) \in E(G \circ H)$ if and only if $u x \in E(G)$ or $u=x$ and $v y \in E(H)$. Notice that for any $u \in V(G)$ the subgraph of $G \circ H$ induced by $\{u\} \times V(H)$ is isomorphic to $H$. For simplicity, we will denote this subgraph by $H_{u}$.

For a basic introduction to the lexicographic product of two graphs we suggest the books $[11,13]$. One of the main problems in the study of $G \circ$ $H$ consists of finding exact values or tight bounds of specific parameters of these graphs and express them in terms of known invariants of $G$ and $H$. In particular, we cite the following works on domination theory of lexicographic product graphs: (total) domination [20,23,30], Roman domination [27], weak Roman domination [29], rainbow domination [28], super domination [8], doubly connected domination [1], secure domination [16], double domination [3] and total Roman domination $[4,6]$.

The following claim, which states the distance formula in the lexicographic product of two graphs, is one of our main tools.

Remark 1. [11] For any connected graph $G$ of order $\mathrm{n}(G) \geq 2$ and any graph $H$, the following statements hold.

(i) $d_{G \circ H}\left((g, h),\left(g^{\prime}, h^{\prime}\right)\right)=d_{G}\left(g, g^{\prime}\right)$ for $g \neq g^{\prime}$.

(ii) $d_{G \circ H}\left((g, h),\left(g, h^{\prime}\right)\right)=\min \left\{2, d_{H}\left(h, h^{\prime}\right)\right\}$ for $g=g^{\prime}$.

Given a set $W \subseteq V(G) \times V(H)$, the projection of $W$ on $V(G)$ will be denoted by $P_{G}(W)$. The following corollary is a direct consequence of the previous remark.

Corollary 5. Let $G$ be a connected graph of order $\mathrm{n}(G) \geq 2$ and let $H$ be a graph. A set $W \subseteq V(G) \times V(H)$ is a 2-packing of $G \circ H$ if and only if $P_{G}(W)$ is a 2-packing of $G$ and $|W|=\left|P_{G}(W)\right|$.

In the following result we show general lower and upper bounds for the 2-packing differential of a lexicographic product graph, in terms of some parameters of both factors.

We define the following parameter.

$$
\rho^{\prime}(G)=\max \left\{|S|: S \text { is a } \partial_{2 p}(G) \text {-set }\right\} .
$$

Theorem 17. For any connected graph $G$ of order $\mathrm{n}(G) \geq 2$ and any graph $H$,

$$
\begin{aligned}
& \mathrm{n}(H) \partial_{2 p}(G)+\rho^{\prime}(G)(\mathrm{n}(H)+\Delta(H)-1) \leq \partial_{2 p}(G \circ H) \leq \mathrm{n}(H) \partial_{2 p}(G) \\
& \quad+\rho(G)(\mathrm{n}(H)+\Delta(H)-1) .
\end{aligned}
$$


Furthermore,

$$
\begin{aligned}
& \mathrm{n}(H) \mu_{R}(G)-\rho(G)(\mathrm{n}(H)+\Delta(H)-1) \leq \mu_{R}(G \circ H) \leq \mathrm{n}(H) \mu_{R}(G) \\
& \quad-\rho^{\prime}(G)(\mathrm{n}(H)+\Delta(H)-1) .
\end{aligned}
$$

Proof. Let $W \subseteq V(G) \times V(H)$ be a $\partial_{2 p}(G \circ H)$-set and $U=P_{G}(W)$. By Corollary 5, we learned that $U$ is a 2-packing of $G$. Hence,

$$
\begin{aligned}
\partial_{2 p}(G \circ H) & =|N(W)|-|W| \\
& =\sum_{(g, h) \in W}|N(g)| \mathrm{n}(H)+\sum_{(g, h) \in W} \operatorname{deg}(h)-|W| \\
& \leq|N(U)| \mathrm{n}(H)+|U| \Delta(H)-|U| \\
& =\partial(U) \mathrm{n}(H)+|U|(\mathrm{n}(H)+\Delta(H)-1) \\
& \left.\leq \partial_{2 p}(G) \mathrm{n}(H)+\rho(G)(\mathrm{n}(H)+\Delta(H)-1)\right) .
\end{aligned}
$$

Now, for any $\partial_{2 p}(G)$-set $S$ with $|S|=\rho^{\prime}(G)$ and any $v \in V(H)$ with $\operatorname{deg}(v)=$ $\Delta(H)$, the set $S \times\{v\}$ is a 2-packing of $G \circ H$, and so

$$
\begin{aligned}
\partial_{2 p}(G \circ H) & \geq \partial(S \times\{v\}) \\
& =\sum_{u \in S}(|N(u)| \mathrm{n}(H)+\Delta(H))-\rho^{\prime}(G) \\
& =|N(S)| \mathrm{n}(H)+\Delta(H) \rho^{\prime}(G)-\rho^{\prime}(G) \\
& =\partial(S) \mathrm{n}(H)+\rho^{\prime}(G)(\mathrm{n}(H)+\Delta(H)-1) \\
& =\partial_{2 p}(G) \mathrm{n}(H)+\rho^{\prime}(G)(\mathrm{n}(H)+\Delta(H)-1) .
\end{aligned}
$$

By Theorem 15 we complete the proof.

If $G$ is a regular graph, then $\rho(G)=\rho^{\prime}(G)$. Hence, from Theorem 17 we derive the following result.

Corollary 6. The following statements hold for any connected regular graph $G$ of order $\mathrm{n}(G) \geq 2$ and any graph $H$.

- $\partial_{2 p}(G \circ H)=\mathrm{n}(H) \partial_{2 p}(G)+\rho(G)(\mathrm{n}(H)+\Delta(H)-1)$.

- $\mu_{R}(G \circ H)=\mathrm{n}(H) \mu_{R}(G)-\rho(G)(\mathrm{n}(H)+\Delta(H)-1)$.

If $G$ is an efficient closed domination graph with $\partial_{2 p}(G)=\mathrm{n}(G)-2 \rho(G)$, then $\rho(G)=\rho^{\prime}(G)$. Therefore, Theorem 17 leads to the following result.

Corollary 7. If $G$ is a connected efficient closed domination graph with $\mathrm{n}(G) \geq$ 2 and $\partial_{2 p}(G)=\mathrm{n}(G)-2 \rho(G)$, then the following statements hold for any graph $H$,

$$
\begin{aligned}
& -\partial_{2 p}(G \circ H)=\mathrm{n}(H) \partial_{2 p}(G)+\rho(G)(\mathrm{n}(H)+\Delta(H)-1) . \\
& -\mu_{R}(G \circ H)=\mathrm{n}(H) \mu_{R}(G)-\rho(G)(\mathrm{n}(H)+\Delta(H)-1) .
\end{aligned}
$$

We finish with the computation of the exact values of the 2-packing differential and the unique response Roman domination number of the lexicographic product of a path and any other graph. 
Theorem 18. Let $r \geq 2$ be an integer. If $H$ is a nonempty graph, then

$$
\partial_{2 p}\left(P_{r} \circ H\right)=\left\{\begin{array}{lll}
\frac{r}{3}(2 \mathrm{n}(H)+\Delta(H)-1), & r \equiv 0 & (\bmod 3), \\
\frac{2}{3}(r-1) \mathrm{n}(H)+\frac{r+2}{3}(\Delta(H)-1), r \equiv 1 & (\bmod 3), \\
\frac{2 r-1}{3} \mathrm{n}(H)+\frac{r+1}{3}(\Delta(H)-1), & r \equiv 2 & (\bmod 3) .
\end{array}\right.
$$

Furthermore,

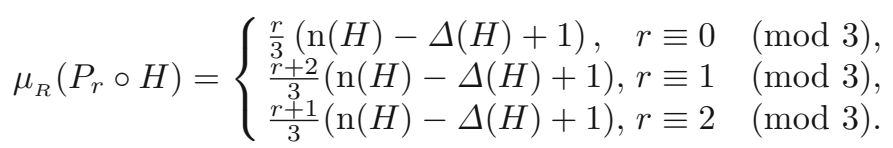

Proof. If $r \equiv 0,2(\bmod 3)$, then $\rho^{\prime}\left(P_{r}\right)=\rho\left(P_{r}\right)$. Hence, the result follows from Theorem 17 . Now, assume $r \equiv 1(\bmod 3)$, and let $S$ be a $\rho\left(P_{r}\right)$-set. In this case, $P_{r}$ is an efficient closed domination graph, and so $|N(S)|=r-|S|=\frac{2(r-1)}{3}$. Thus, for every vertex $v \in V(H)$ with $\operatorname{deg}(v)=\Delta(H)$,

$$
\begin{aligned}
\partial_{2 p}\left(P_{r} \circ H\right) & \geq \partial(S \times\{v\}) \\
& =\sum_{u \in S}(|N(u)| \mathrm{n}(H)+\Delta(H)-1) \\
& =|N(S)| \mathrm{n}(H)+\rho\left(P_{r}\right)(\Delta(H)-1) \\
& =\frac{2}{3}(r-1) \mathrm{n}(H)+\frac{r+2}{3}(\Delta(H)-1) .
\end{aligned}
$$

It remains to show that the equality holds. To this end, let $W$ be a $\partial_{2 p}\left(P_{r} \circ H\right)$ set and $U$ the projection of $W$ on $V\left(P_{r}\right)$. By Corollary 5 , we learned that $U$ is a 2-packing of $P_{r}$. Hence, as in the proof of Theorem 17 we deduce that $\partial_{2 p}\left(P_{r} \circ H\right) \leq|N(U)| \mathrm{n}(H)+|U|(\Delta(H)-1)$. Thus, if $U$ is a $\rho\left(P_{r}\right)$-set, then $\partial_{2 p}\left(P_{r} \circ H\right)=\frac{2}{3}(r-1) \mathrm{n}(H)+\frac{r+2}{3}(\Delta(H)-1)$, as required. Now, suppose to the contrary, that $|U| \leq \rho\left(P_{r}\right)-1=\frac{r-1}{3}$. In such a case, $|N(U)| \leq 2|U| \leq \frac{2(r-1)}{3}$, and so

$$
\begin{aligned}
\frac{2}{3}(r-1) \mathrm{n}(H)+\frac{r+2}{3}(\Delta(H)-1) & \leq \partial_{2 p}\left(P_{r} \circ H\right) \\
& \leq|N(U)| \mathrm{n}(H)+|U|(\Delta(H)-1) \\
& \leq \frac{2}{3}(r-1) \mathrm{n}(H)+\frac{r-1}{3}(\Delta(H)-1),
\end{aligned}
$$

which is a contradiction for $\Delta(H) \geq 2$, and we have equalities for $\Delta(H)=1$. By Theorem 15 we complete the proof.

We can make the appropriate modifications in the proof of the previous theorem to show that the following result follows.

Theorem 19. Let $r \geq 2$ be an integer. If $H$ is an empty graph, then

$$
\partial_{2 p}\left(P_{r} \circ H\right)=\left\{\begin{array}{lll}
\frac{r}{3}(2 \mathrm{n}(H)-1), & r \equiv 0 & (\bmod 3), \\
\frac{r-1}{3}(2 \mathrm{n}(H)-1), & r \equiv 1 & (\bmod 3), \\
\frac{2 r-1}{3} \mathrm{n}(H)-\frac{r+1}{3}, r \equiv 2 & (\bmod 3) .
\end{array}\right.
$$


Furthermore,

$$
\mu_{R}\left(P_{r} \circ H\right)=\left\{\begin{array}{lll}
\frac{r}{3}(\mathrm{n}(H)+1), & r \equiv 0 & (\bmod 3), \\
\frac{r+2}{3} \mathrm{n}(H)+\frac{r-1}{3}, & r \equiv 1 & (\bmod 3), \\
\frac{r+1}{3}(\mathrm{n}(H)+1), & r \equiv 2 & (\bmod 3) .
\end{array}\right.
$$

Author contributions All the authors participated in all stages of this paper.

Funding Open Access funding provided thanks to the CRUE-CSIC agreement with Springer Nature.

\section{Declaration}

Conflict of interest The authors declare no conflict of interest

Open Access. This article is licensed under a Creative Commons Attribution 4.0 International License, which permits use, sharing, adaptation, distribution and reproduction in any medium or format, as long as you give appropriate credit to the original author(s) and the source, provide a link to the Creative Commons licence, and indicate if changes were made. The images or other third party material in this article are included in the article's Creative Commons licence, unless indicated otherwise in a credit line to the material. If material is not included in the article's Creative Commons licence and your intended use is not permitted by statutory regulation or exceeds the permitted use, you will need to obtain permission directly from the copyright holder. To view a copy of this licence, visit http://creativecommons. org/licenses/by/4.0/.

\section{References}

[1] Arriola, B.H., Canoy Jr., S.R.: Doubly connected domination in the corona and lexicographic product of graphs. Appl. Math. Sci. (Ruse) 8(29-32), 1521-1533 (2014). https://doi.org/10.12988/ams.2014.4136

[2] Bermudo, S., Hernández-Gómez, J.C., Rodríguez, J.M., Sigarreta, J.M.: Relations between the differential and parameters in graphs. Electron. Notes Discrete Math. 46, 281-288 (2014). https://doi.org/10.1016/j.endm.2014.08.037

[3] Cabrera Martínez, A., Cabrera García, S., Rodríguez-Velázquez, J.A.: Double domination in lexicographic product graphs. Discrete Appl. Math. 284, 290-300 (2020). https://doi.org/10.1016/j.dam.2020.03.045

[4] Cabrera Martínez, A., Rodríguez-Velázquez, J.A.: Closed formulas for the total Roman domination number of lexicographic product graphs. Ars Math. Contemp. (2020). https://doi.org/10.26493/1855-3974.2284.aeb

[5] Cabrera Martínez, A., Rodríguez-Velázquez, J.A.: On the perfect differential of a graph. Quaest. Math. (2021). https://doi.org/10.2989/16073606.2020.1858992

[6] Campanelli, N., Kuziak, D.: Total Roman domination in the lexicographic product of graphs. Discrete Appl. Math. 263, 88-95 (2019). https://doi.org/10.1016/ j.dam.2018.06.008 
[7] Cockayne, E.J., Dreyer Jr., P.A., Hedetniemi, S.M., Hedetniemi, S.T.: Roman domination in graphs. Discrete Math. 278(1-3), 11-22 (2004). https://doi.org/ 10.1016/j.disc.2003.06.004

[8] Dettlaff, M., Lemańska, M., Rodríguez-Velázquez, J.A., Zuazua, R.: On the super domination number of lexicographic product graphs. Discrete Appl. Math. 263, 118-129 (2019). https://doi.org/10.1016/j.dam.2018.03.082

[9] Ebrahimi Targhi, E., Jafari Rad, N., Volkmann, L.: Unique response Roman domination in graphs. Discrete Appl. Math. 159(11), 1110-1117 (2011). https:// doi.org/10.1016/j.dam.2011.03.013

[10] Goddard, W., Henning, M.A.: Generalised domination and independence in graphs. Congr. Numer. 123, 161-171 (1997)

[11] Hammack, R., Imrich, W., Klavžar, S.: Handbook of Product Graphs, Discrete Mathematics and Its Applications, 2nd edn. CRC Press, Boca Raton (2011)

[12] Haynes, T.W., Hedetniemi, S.T., Slater, P.J.: Fundamentals of Domination in Graphs, Chapman and Hall/CRC Pure and Applied Mathematics Series. Marcel Dekker, Inc., New York (1998)

[13] Imrich, W., Klavžar, S.: Product Graphs, Structure and Recognition. WileyInterscience Series in Discrete Mathematics and Optimization. Wiley, New York (2000)

[14] Jafari Rad, N., Liu, C.H.: Trees with strong equality between the Roman domination number and the unique response Roman domination number. Australas. J. Combin. 54, 133-140 (2012)

[15] Klavžar, S., Peterin, I., Yero, I.: Graphs that are simultaneously efficient open domination and efficient closed domination graphs. Discrete Appl. Math. 217(3), 613-621 (2017). https://doi.org/10.1016/j.dam.2016.09.027

[16] Klein, D.J., Rodríguez-Velázquez, J.A.: Protection of lexicographic product graphs. Discuss. Math. Graph Theory (2020). https://doi.org/10.7151/dmgt. 2243

[17] Kong, M.C., Zhao, Y.: On computing maximum $k$-independent sets. In: Proceedings of the Twenty-Fourth Southeastern International Conference on Combinatorics, Graph Theory, and Computing, vol. 95, pp. 47-60. Boca Raton (1993)

[18] Kuziak, D., Peterin, I., Yero, I.G.: Efficient open domination in graph products. Discrete Math. Theor. Comput. Sci. 16(1), 105-120 (2014)

[19] Lewis, J.L., Haynes, T.W., Hedetniemi, S.M., Hedetniemi, S.T., Slater, P.J.: Differentials in graphs. Util. Math. 69, 43-54 (2006)

[20] Liu, J., Zhang, X., Meng, J.: Domination in lexicographic product digraphs. Ars Combin. 120, 23-32 (2015)

[21] Meir, A., Moon, J.W.: Relations between packing and covering numbers of a tree. Pac. J. Math. 61(1), 225-233 (1975). https://doi.org/10.2140/pjm.1975. 61.225

[22] Nordhaus, E.A., Gaddum, J.W.: On complementary graphs. Am. Math. Mon. 63, 175-177 (1956). https://doi.org/10.2307/2306658

[23] Nowakowski, R.J., Rall, D.F.: Associative graph products and their independence, domination and coloring numbers. Discuss. Math. Graph Theory 16, 5379 (1996). https://doi.org/10.7151/dmgt.1023 
[24] Pushpam, P.R.L., Yokesh, D.: Differentials in certain classes of graphs. Tamkang J. Math. 41(2), 129-138 (2010). https://doi.org/10.5556/j.tkjm.41.2010.664

[25] Rubalcaba, R.R., Slater, P.J.: Roman dominating influence parameters. Discrete Math. 307(24), 3194-3200 (2007). https://doi.org/10.1016/j.disc.2007.03.020

[26] Sigarreta, J.M.: Differential in Cartesian product graphs. Ars Combin. 126, 259267 (2016)

[27] Šumenjak, T.K., Pavlič, P., Tepeh, A.: On the Roman domination in the lexicographic product of graphs. Discrete Appl. Math. 160(13-14), 2030-2036 (2012). https://doi.org/10.1016/j.dam.2012.04.008

[28] Šumenjak, T.K., Rall, D.F., Tepeh, A.: Rainbow domination in the lexicographic product of graphs. Discrete Appl. Math. 161(13-14), 2133-2141 (2013). https:// doi.org/10.1016/j.dam.2013.03.011

[29] Valveny, M., Pérez-Rosés, H., Rodríguez-Velázquez, J.A.: On the weak Roman domination number of lexicographic product graphs. Discrete Appl. Math. 263, 257-270 (2019). https://doi.org/10.1016/j.dam.2018.03.039

[30] Zhang, X., Liu, J., Meng, J.: Domination in lexicographic product graphs. Ars Combin. 101, 251-256 (2011)

[31] Zhao, N., Li, W., Zhao, T., Zhang, Z.: The unique response Roman domination in trees. J. Combin. Math. Combin. Comput. 105, 165-183 (2018)

A. Cabrera Martínez and J. A. Rodríguez-Velázquez

Departament d'Enginyeria Informàtica i Matemàtiques

Universitat Rovira i Virgili

Av. Països Catalans 26

43007 Tarragona

Spain

e-mail: abel.cabrera@urv.cat;

juanalberto.rodriguez@urv.cat

M. L. Puertas

Departamento de Matemáticas

Universidad de Almería

Carretera Sacramento s/n

04120 Almería

Spain

e-mail: mpuertas@ual.es

Received: February 13, 2021.

Accepted: July 8, 2021.

Publisher's Note Springer Nature remains neutral with regard to jurisdictional claims in published maps and institutional affiliations. 